Pregnancy and systemic sclerosis

A study of pregnancy in this condition showed that there was no increase in either the risk of miscarriage or perinatal death compared with controls. Preterm births though were slightly more prevalent and the babies tended to be smaller. The authors warn, however, that where there is early and rapidly progressive, diffuse skin thickening pregnancy should be avoided because of an increased risk of renal disease.

Arthritis Rheum 1989; 32: 151-7.

\section{Clinical trials and GCP}

Good Clinical Practice (GCP) is the name applied to the format for collecting the data for clinical drug trial studies these days. It looks as if the drug companies may insist on the use of this format increasingly for clinical drug research on patients. The procedure is complex and time consuming and therefore costly, and it involves much closer monitoring of such trials by ethical committees. You have been warned. Lancet 1989; i: 1008-9.

\section{Salami papers}

Arnold Relman, the editor-in-chief of the New England Fournal of Medicine, again draws attention to the tendency for some investigators to publish the information arising from a particular study in more than one paper, producing the evidence in sections, though with some overlap. This is the salami syndrome. The idea of course is to maximise the number of papers published by the department. The result is to confuse readers and editors alike and is wasteful of resources at a time when there is increasing pressure on scientific journals. Naturally there is on occasion a good reason for producing the work this way, but too often there is not. The increased emphasis on quality rather than quantity when judging the output of a given department may modify this practice for the future.

$N$ Engl f Med 1989; 320: 1212-4.

Familial osteoarthritis and type II collagen gene Osteoarthritis is proving to be an increasingly happy hunting ground for primary research and the identification of subsets is now becoming more sophisticated. Two families are studied in this report with familial premature osteoarthritis where a linkage has been discovered between the disease and a type II collagen gene on chromosome 12 by use of restriction fragment length polymorphisms. The inheritance pattern was autosomal dominant with virtually complete penetrance.

Lancet 1989; i: 924-7.

\section{Backache, stress, and lifestyle}

Low back pain is one of the scourges of society and is the source of an immense amount of loss of time from work and attendance at doctors' surgeries and hospitals as a result. This Swedish study of 1760 women showed that the lifetime incidence was $66 \%$ with a prevalence of $35 \%$. Many work factors seemed to correlate with it, including bending, lifting, and standing. Analysis, however, suggested that the only variables directly associated with low back pain were dissatisfaction with the work environment, a higher degree of worry, and fatigue at the end of the day.

Spine 1989; 14: 517-22.

Rheumatoid arthritis, TNF alpha antibodies and interleukin 1 production

Interleukin 1 (ILl) and tumour necrosis factor (TNF alpha) occur in the synovial fluid in rheumatoid arthritis, and the cytokines are very active in inducing both bone and cartilage damage. Anti-TNF alpha antibody significantly reduced production of synovial ILl in rheumatoid arthritis but this cytokine was only produced in small amounts in osteoarthritis, and anti-TNF alpha antibody had no effect on its production. This effect it is suggested may be a useful addition to the methods available for treating rheumatoid arthritis. The difference observed between the two diseases may suggest that other factors are involved in ILl induction in rheumatoid arthritis but not osteoarthritis.

Lancet 1989; ii: 244-7.

\section{Wrist joint loading}

Little study has been made of the effect of pressure distribution and the contact area in the human wrist joint. An experiment on cadaveric wrists showed no difference in loading whether it was distributed through two or five metacarpals, and there was a non-linear relation between increasing loads and increase in contact areas. More loading seemed to occur through the scaphoid rather than the lunate area, and even at the highest loading no more than $40 \%$ of the available surface area of the wrist was in contact, reflecting the incongruence of the joint.

f Hand Surg [Am] 1989; 14: 458-65.

When to examine aspirated synovial fluid

The answer to this seems to be the same day. The white cell count drops within a few hours and calcium pyrophosphate crystals become difficult to recognise by the next day as they rapidly dissolve. Monosodium urate crystals fare better, as do those of hydroxyapatite, but many artefacts appear, such as Maltese cross forms, and interpretation rapidly becomes difficult. The motto is-do it quickly after aspiration. Arthritis Rhem 1989; 32: 271-8.

\section{Paget's disease and arthrodesis of the knee}

There have apparently been no previous reports of monostotic Paget's disease crossing the site of fusion into previously unaffected bone. This was, however, observed after arthrodesis of the knee in a 47 year old Canadian. The disease extended centrifugally from the medullary canal to the cortical bone as has previously been observed. f Bone foint Surg [Am] 1989; 71: 129-32.

GEMAENNES 